\title{
Remark on the effective potential of the gravitational perturbation in the black hole background projected on the brane
}

\author{
D. K. Park* \\ Department of Physics, Kyungnam University, Masan, 631-701, Korea.
}

(July 19, 2018)

\begin{abstract}
The polar perturbation is examined when the spacetime is expressed by a $4 d$ metric induced from higher-dimensional Schwarzschild geometry. Since the spacetime background is not a vacuum solution of $4 d$ Einstein equation, the various general principles are used to understand the behavior of the energymomentum tensor under the perturbation. It is found that although the general principles fix many components, they cannot fix two components of the energy-momentum tensor. Choosing two components suitably, we derive the effective potential which has a correct $4 d$ limit.
\end{abstract}

*Email:dkpark@hep.kyungnam.ac.kr 
The spacetime stability/instability problem is an important issue to determine the final state of the gravitational collapse. It is also important in the astrophysical side for the detection of the gravitational wave. Long ago this issue was firstly examined by Regge and Wheeler [1] in the background of the $4 d$ Schwarzschild geometry. In four spacetime dimension there are two types of the gravitational perturbations called axial (or odd-parity) and polar (or even-parity) perturbations [2]. The Schwarzschild geometry in general admits the perturbed equations to be separable and the resultant radial equations can be transformed into the Schrödinger-like form. Thus the effective potential for each perturbation was explicitly derived in Ref. [3,4]. The transformation into the Schrödinger-like expression is important to predict the various physical phenomena because we have much background in quantum mechanics.

Recent quantum gravity such as string theories [5] and the brane-world scenarios $[6,7]$ in general introduce the extra dimensions to reconcile general relativity with quantum physics. In this context, recently, much attention is paid to the higher-dimensional spacetime. The various spacetime metrics of the higher-dimensional black holes were derived in Ref. [8,9]. The gravitational perturbations in the higher-dimensional Schwarzschild background were also discussed in Ref. [10]. When the spacetime dimension is more than four, there are three types of the gravitational perturbations called scalar, vector, and tensor perturbations. The scalar and vector perturbations correspond to the polar and the axial perturbations in four dimension. There is no correspondence of the tensor perturbation in four dimension.

Recently, the axial perturbation is discussed when the spacetime is a $4 d$ metric induced from the higher-dimensional Schwarzschild black hole [11]. In the following we will briefly review Ref. [11]. Subsequently the polar perturbation will be addressed in this background.

We start with an $4 d$ metric which is induced from the $(4+n)$-dimensional Schwarzschild black hole

$$
d s^{2}=-h(r) d t^{2}+h^{-1}(r) d r^{2}+r^{2}\left(d \theta^{2}+\sin ^{2} \theta d \phi^{2}\right)
$$

where $h(r)=1-\left(r_{H} / r\right)^{n+1}$. As mentioned, there are two types of the gravitational metric 
perturbations in four spacetime dimension called axial and polar perturbations $[1,2]$. In the former case the metric is changed into $d s^{2}+\delta s_{A}^{2}$ where

$$
\delta s_{A}^{2}=\left[H_{0}(r) d t d \phi+H_{1}(r) d r d \phi\right] e^{i \omega t} \sin \theta \frac{d P_{\ell}}{d \theta}(\cos \theta)
$$

while the metric for the latter case is $d s^{2}+\delta s_{P}^{2}$ where

$$
\delta s_{P}^{2}=\left[H(r) h d t^{2}+H(r) h^{-1} d r^{2}+r^{2} K(r)\left(d \theta^{2}+\sin ^{2} \theta d \phi^{2}\right)+2 H_{1}(r) d t d r\right] e^{i \omega t} P_{\ell}(\cos \theta)
$$

where $P_{\ell}(\cos \theta)$ is an usual Legendre polynomial. The various $r$-dependent functions are assumed to be small for the linearization.

When $n=0$, the metric in Eq.(1) is an usual $4 d$ Schwarzschild metric which is a vacuum solution of the Einstein equation. In this $4 d$ case, therefore, the gravitational linearized fluctuation can be expressed as $\delta R_{\mu \nu}=0$ where $R_{\mu \nu}$ is a Ricci tensor. Using this representation the effective potentials for the perturbations were derived long ago $[3,4]$

$$
\begin{aligned}
& V_{A}(r)=h\left[\frac{2 \lambda+2}{r^{2}}-\frac{3}{r^{2}}\left(\frac{r_{H}}{r}\right)\right] \\
& V_{P}(r)=\frac{h}{\left(2 \lambda r+3 r_{H}\right)^{2}}\left[8 \lambda^{2}(\lambda+1)+12+12 \lambda^{2}\left(\frac{r_{H}}{r}\right)+18 \lambda\left(\frac{r_{H}}{r}\right)^{2}+9\left(\frac{r_{H}}{r}\right)^{3}\right]
\end{aligned}
$$

where $\lambda=(\ell-1)(\ell+2) / 2$.

The most interesting feature of the potentials is that they are related to each other as following $[2]$

$$
V_{P, A}(r)= \pm \beta \frac{d f}{d r_{*}}+\beta^{2} f^{2}+\kappa f
$$

where the upper(lower) sign corresponds to the polar(axial) perturbation and $r_{*}$ is a "tortoise" coordinate defined $d r / d r_{*}=h$. In Eq.(5) $\beta=3 r_{H}, \kappa=4 \lambda(\lambda+1)$ and

$$
f=\frac{h}{r\left(2 \lambda r+3 r_{H}\right)}
$$

In fact this relation was found when the Newman-Penrose formalism [12] is applied to the gravitational perturbations. This explicit relation between $V_{P}$ and $V_{A}$ enables us to realize 
that both potentials have the same transmission coefficient ${ }^{1}$.

In this context the following several questions arise naturally: (i) Do the effective potentials exist when the spacetime is a metric induced from the higher-dimensional Schwarzschild black hole? (ii) If the potentials exist, is there any relation between them like Eq.(5)? (iii) Do they have same transmission coefficient like $4 d$ case? We would like to address the first issue as much as possible in this letter.

The most difficult problem one copes with when the spacetime is induced from the higher-dimensional one is the fact that Eq.(1) is not a vacuum solution of the $4 d$ Einstein equation if $n \neq 0$. Thus what we can do is to assume that Eq.(1) is a non-vacuum solution, i.e. $\mathcal{E}_{\mu \nu}=T_{\mu \nu}$ where $\mathcal{E}_{\mu \nu}$ and $T_{\mu \nu}$ are Einstein and energy-momentum tensors, respectively. Since, however, $T_{\mu \nu}$ is not originated from the real matter living on the brane, but appears effectively in the course of the projection of the bulk metric to the brane, we do not know how $T_{\mu \nu}$ is transformed when the perturbations (2) or (3) is turned on. The only way, in our opinion, to get rid of this difficulty is to get an information on the energy-momentum tensor as much as possible from the general principles.

The fluctuation equation in the background of the non-vacuum solution is generally expressed as $\delta \mathcal{E}_{\mu \nu}=\delta T_{\mu \nu}$. Thus the difficulty mentioned above is how to obtain $\delta T_{\mu \nu}$ from the general principles without knowing the exact nature of the matter.

The axial perturbation in the background of Eq.(1) was discussed in Ref. [11]. In this case the non-vanishing components of $\delta T_{\mu \nu}$ are $\delta T_{t \phi}, \delta T_{r \phi}$ and $\delta T_{\theta \phi}$. The general principles used in Ref. [11]: (i) covariant conservation of $T^{\mu \nu}$ (ii) linear dependence of the Einstein equation (iii) the correct $4 d$ limit of the effective potential. It turned out that the first principle generates the unique non-trivial constraint to the energy-momentum tensor. But

\footnotetext{
${ }^{1}$ In Ref. [2] the general criterion for the same transmission coefficient of the different onedimensional potentials was derived in terms of the $\mathrm{KdV}$ equation, which is well-known in the solitonic theories.
} 
the second one is automatically satisfied if the first principle is used. Third one makes a constraint only in the $4 d$ limit of the energy-momentum tensor. Thus the principle used in Ref. [11] could not completely determine $\delta T_{t \phi}, \delta T_{r \phi}$ and $\delta T_{\theta \phi}$. Thus what we can do in this situation is to choose the energy-momentum tensor consistently with the general principles. In Ref. [11] $\delta T_{r \phi}=\delta T_{\theta \phi}=0$ is chosen for simplicity. Then the effective potential for the axial perturbation becomes

$$
V_{A}(r)=h(r)\left[\frac{2 \lambda+2}{r^{2}}-\left\{(n+1)^{2}+2\right\}\left(\frac{r_{H}}{r}\right)^{n+1}\right] .
$$

It is easy to show that Eq.(7) has a correct $4 d$ limit. But the problem is that the different choice may yield a different potential.

Now we would like to consider the polar perturbation by adding the perturbation (3) into (1). From the non-vanishing components of $\delta \mathcal{E}_{\mu \nu}$ in the gravity side it is reasonable to assume that the non-vanishing components of $\delta T_{\mu \nu}$ are $\delta T_{t t}, \delta T_{t r}, \delta T_{t \theta}, \delta T_{r r}, \delta T_{r \theta}, \delta T_{\theta \theta}$ and $\delta T_{\phi \phi}$. The spherical symmetry of course guarantees $\delta T_{\phi \phi}=\delta T_{\theta \theta} \sin ^{2} \theta$.

The $(t, r),(t, \theta)$ and $(r, \theta)$ componets of the Einstein equations reduce to

$$
\begin{aligned}
K^{\prime} & =\frac{i}{\omega} \delta \Theta_{t r}+\left(\frac{h^{\prime}}{2 h}-\frac{1}{r}\right) K-\frac{i}{\omega}\left(\frac{h^{\prime}}{r}+\frac{h+\lambda}{r^{2}}\right) H_{1}+\frac{1}{r} H \\
H_{1}^{\prime} & =\frac{2}{h} \delta \Theta_{t \theta}-\frac{h^{\prime}}{h} H_{1}+\frac{i \omega}{h}(H+K) \\
H^{\prime} & =\frac{i}{\omega} \delta \Theta_{t r}+2 \delta \Theta_{r \theta}+\left(\frac{1}{r}-\frac{h^{\prime}}{h}\right) H+\left(\frac{h^{\prime}}{2 h}-\frac{1}{r}\right) K-\frac{i}{\omega}\left(\frac{h^{\prime}}{r}+\frac{h+\lambda}{r^{2}}-\frac{\omega^{2}}{h}\right) H_{1}
\end{aligned}
$$

where the prime denotes a differentiation with respect to $r$ and

$$
\begin{aligned}
\delta T_{t r} & =\delta \Theta_{t r}(r) e^{i \omega t} P_{\ell}(\cos \theta) \\
\delta T_{t \theta} & =\delta \Theta_{t \theta}(r) e^{i \omega t} \frac{d P_{\ell}}{d \theta}(\cos \theta) \\
\delta T_{r \theta} & =\delta \Theta_{r \theta}(r) e^{i \omega t} \frac{d P_{\ell}}{d \theta}(\cos \theta) .
\end{aligned}
$$

Now we consider the covariant conservation of the energy-momentum tensor, i.e. $T_{; \mu}^{\mu \nu}=$ 0 . This gives three conditions when $\nu=t, r$, and $\theta$. Using the conditions derived when $\nu=t$ and $\theta$, one can express $\delta T_{t t}$ and $\delta T_{\theta \theta}$ in terms of the non-diagonal components as following: 


$$
\begin{aligned}
\delta T_{t t}= & -\frac{i}{\omega}\left[h^{2}\left\{\partial_{r} \delta \Theta_{t r}+\left(\frac{2}{r}+\frac{h^{\prime}}{h}\right) \delta \Theta_{t r}\right\}-\frac{2 h}{r}\left(\frac{h+\lambda}{r}+h^{\prime}\right) \delta \Theta_{t \theta}\right. \\
\left.-\frac{h^{2}}{r}\left(h^{\prime}+\frac{r}{2} h^{\prime \prime}\right)\left(\frac{i \omega}{h} K+\frac{2}{r} H_{1}\right)\right] e^{i \omega t} P_{\ell}(\cos \theta) & \\
\delta T_{\theta \theta}= & {\left[\frac{i \omega r^{2}}{h} \delta \Theta_{t \theta}-r^{2} h\left\{\partial_{r} \delta \Theta_{r \theta}+\left(\frac{h^{\prime}}{h}+\frac{2}{r}\right) \delta \Theta_{r \theta}\right\}+r\left(h^{\prime}+\frac{r}{2} h^{\prime \prime}\right) K\right] e^{i \omega t} P_{\ell}(\cos \theta) . }
\end{aligned}
$$

With an aid of Eq.(10) the condition at $\nu=r$ gives the following differential equation for $\delta T_{r r}:$

$$
\partial_{r} \delta T_{r r}+\left(\frac{3 h^{\prime}}{2 h}+\frac{2}{r}\right) \delta T_{r r}=\left(Q_{1}(r)+Q_{2}(r)\right) e^{i \omega t} P_{\ell}(\cos \theta)
$$

where

$$
\begin{aligned}
Q_{1}(r)= & \frac{i}{\omega} \frac{h^{\prime}}{2 h}\left[\partial_{r} \delta \Theta_{t r}+\left(\frac{4}{r}+\frac{h^{\prime}}{h}+\frac{h^{\prime \prime}}{h^{\prime}}+\frac{2 \omega^{2}}{h h^{\prime}}\right) \delta \Theta_{t r}\right] \\
& -\frac{2}{r}\left[\partial_{r} \delta \Theta_{r \theta}+\frac{h-\lambda}{r h} \delta \Theta_{r \theta}\right]-\frac{i}{\omega} \frac{1}{r h^{2}}\left[\frac{(h+\lambda) h^{\prime}}{r}+h^{\prime 2}-2 \omega^{2}\right] \delta \Theta_{t \theta} \\
Q_{2}(r)= & \left(h^{\prime}+\frac{r}{2} h^{\prime \prime}\right)\left[\frac{3}{r^{2} h} H-\frac{i}{\omega r h}\left(\frac{2 h^{\prime}}{r}+\frac{h+\lambda}{r^{2}}\right) H_{1}+\frac{1}{r h}\left(\frac{h^{\prime}}{h}-\frac{1}{r}\right) K\right] .
\end{aligned}
$$

Thus $\delta T_{r r}$ also can be obtained from the non-diagonal components of the energy-momentum tensor as following:

$$
\delta T_{r r}=\frac{1}{r^{2} h^{3 / 2}} e^{i \omega t} P_{\ell}(\cos \theta) \int d r\left(Q_{1}+Q_{2}\right) r^{2} h^{3 / 2}
$$

Therefore all diagonal components can be calculated if $\delta \Theta_{t r}, \delta \Theta_{t \theta}$ and $\delta \Theta_{r \theta}$ are known.

Now, we would like to consider the diagonal components of the Einstein equation. After some algebra one can show straightforwardly that the first-order differential equations in Eq.(8) automatically solve the $(t, t),(\theta, \theta)$ and $(\phi, \phi)$ components of the Einstein equation. The $(r, r)$ component gives a constraint to $\delta T_{r r}$ in a form:

$$
\delta T_{r r}=\delta T_{r r}^{(1)}+\delta T_{r r}^{(2)}
$$

where

$$
\delta T_{r r}^{(1)}=\left(\frac{i h^{\prime}}{2 \omega h} \delta \Theta_{t r}-\frac{2}{r} \delta \Theta_{r \theta}\right) e^{i \omega t} P_{\ell}(\cos \theta)
$$




$$
\begin{aligned}
\delta T_{r r}^{(2)}=\frac{1}{h}\left[\left(\frac{3 h^{\prime}}{2 r}+\frac{\lambda}{r^{2}}\right) H-\right. & \frac{i}{\omega}\left\{\frac{h^{\prime}}{2}\left(\frac{h^{\prime}}{r}+\frac{h+\lambda}{r^{2}}\right)-\frac{\omega^{2}}{r}\right\} H_{1} \\
+ & \left.\left(\frac{h^{\prime 2}}{4 h}-\frac{h^{\prime}}{2 r}+\frac{\omega^{2}}{h}-\frac{\lambda}{r^{2}}\right) K\right] e^{i \omega t} P_{\ell}(\cos \theta) .
\end{aligned}
$$

Combining (11) and (14), one can derive a relation

$$
\frac{i}{\omega}\left(h h^{\prime}+\frac{r h^{\prime 2}}{4}+r \omega^{2}\right) \delta \Theta_{t r}+\left(3 h h^{\prime}+\frac{2 \lambda h}{r}\right) \delta \Theta_{r \theta}-\frac{i}{\omega}\left[\frac{(h+\lambda) h^{\prime}}{r}+h^{\prime 2}-2 \omega^{2}\right] \delta \Theta_{t \theta}=0 .
$$

Thus one of the three non-diagonal components is not linearly independent.

The relation (16) with the first-order differential equations (8) enables us to derive

$$
\frac{d F}{d r}+\frac{h^{\prime}}{2 h} F=\tilde{Q}(r)
$$

where

$$
\begin{aligned}
& F=-\left(3 r h^{\prime}+2 \lambda\right) H+\left[2 i \omega r+\frac{1}{i \omega}\left\{(h+\lambda) h^{\prime}+r h^{\prime 2}\right\}\right] H_{1}+\left(2 \lambda+r h^{\prime}-\frac{r^{2} h^{\prime 2}}{2 h}-\frac{2 r^{2}}{h} \omega^{2}\right) K \\
& \tilde{Q}=-2\left(h^{\prime}+\frac{r}{2} h^{\prime \prime}\right)\left[3 H-\frac{i}{\omega}\left(2 h^{\prime}+\frac{h+\lambda}{r}\right) H_{1}+\left(\frac{r h^{\prime}}{h}-1\right) K\right] .
\end{aligned}
$$

When $n=0, \tilde{Q}$ vanishes and, therefore, $F=0$ is used as an algebraic identity in Ref. [4]. Thus Eq.(17) is an corresponding algebraic identity for our case.

Using $F$ in Eq.(18) one can eliminate $H$ by making use of

$$
\begin{aligned}
& H=\frac{1}{3 r h^{\prime}+2 \lambda}[-F-( 2 i \omega r \\
&\left.+\frac{1}{i \omega}\left\{(h+\lambda) h^{\prime}+r h^{\prime 2}\right\}\right) H_{1} \\
&\left.+\left(2 \lambda+r h^{\prime}-\frac{r^{2} h^{\prime 2}}{2 h}-\frac{2 r^{2} \omega^{2}}{h}\right) K\right] .
\end{aligned}
$$

Then the first-order differential equations for $K$ and $H_{1}$ reduce to

$$
\begin{aligned}
& K^{\prime}=\frac{i}{\omega} \delta \Theta_{t r}-\frac{F}{r\left(3 r h^{\prime}+2 \lambda\right)}+\left[\alpha_{0}(r)+\omega^{2} \alpha_{2}(r)\right] K+\left[\beta_{0}(r)+\omega^{2} \beta_{2}(r)\right] R \\
& R^{\prime}=-\frac{2}{\omega h} \delta \Theta_{t \theta}+\frac{i F}{h\left(3 r h^{\prime}+2 \lambda\right)}+\left[\gamma_{0}(r)+\omega^{2} \gamma_{2}(r)\right] K+\left[\delta_{0}(r)+\omega^{2} \delta_{2}(r)\right] R
\end{aligned}
$$

where $R \equiv-H_{1} / \omega$ and 


$$
\begin{array}{rlrl}
\alpha_{0} & =\frac{h^{\prime}\left(r h^{\prime}-2 h+\lambda\right)}{h\left(3 r h^{\prime}+2 \lambda\right)} & \alpha_{2}=\frac{-2 r}{h\left(3 r h^{\prime}+2 \lambda\right)} & \\
\beta_{0} & =\frac{2 i}{r^{2}\left(3 r h^{\prime}+2 \lambda\right)}\left[r h^{\prime}\left(r h^{\prime}+h\right)+\lambda\left(\lambda+2 r h^{\prime}+h\right)\right] & \beta_{2}=\frac{2 i}{3 r h^{\prime}+2 \lambda} \\
\gamma_{0}=-\frac{i\left(8 \lambda h+8 r h h^{\prime}-r^{2} h^{\prime 2}\right)}{2 h^{2}\left(3 r h^{\prime}+2 \lambda\right)} & \gamma_{2}=\frac{2 i r^{2}}{h^{2}\left(3 r h^{\prime}+2 \lambda\right)} \\
\delta_{0}=-\frac{h^{\prime}\left(4 r h^{\prime}+h+3 \lambda\right)}{h\left(3 r h^{\prime}+2 \lambda\right)} & \delta_{2}=\frac{2 r}{h\left(3 r h^{\prime}+2 \lambda\right)} .
\end{array}
$$

Now, we perform the transformation

$$
\begin{aligned}
K & =f_{1}(r) \hat{K}+g_{1}(r) \hat{R} \\
R & =f_{2}(r) \hat{K}+g_{2}(r) \hat{R} .
\end{aligned}
$$

Then Eq.(20) reads

$$
\begin{gathered}
f_{1} \hat{K}^{\prime}+g_{1} \hat{R}^{\prime}=-\frac{i}{\omega} \delta \Theta_{t r}-\frac{F}{r\left(3 r h^{\prime}+2 \lambda\right)}+\left[-f_{1}^{\prime}+\left(\alpha_{0}+\omega^{2} \alpha_{2}\right) f_{1}+\left(\beta_{0}+\omega^{2} \beta_{2}\right) f_{2}\right] \hat{K} \\
+\left[-g_{1}^{\prime}+\left(\alpha_{0}+\omega^{2} \alpha_{2}\right) g_{1}+\left(\beta_{0}+\omega^{2} \beta_{2}\right) g_{2}\right] \hat{R} \\
f_{2} \hat{K}^{\prime}+g_{2} \hat{R}^{\prime}=\frac{2}{\omega h} \delta \Theta_{t \theta}+\frac{i F}{h\left(3 r h^{\prime}+2 \lambda\right)}+\left[-f_{2}^{\prime}+\left(\gamma_{0}+\omega^{2} \gamma_{2}\right) f_{1}+\left(\delta_{0}+\omega^{2} \delta_{2}\right) f_{2}\right] \hat{K} \\
+\left[-g_{2}^{\prime}+\left(\gamma_{0}+\omega^{2} \gamma_{2}\right) g_{1}+\left(\delta_{0}+\omega^{2} \delta_{2}\right) g_{2}\right] \hat{R} .
\end{gathered}
$$

As in the axial perturbation what can we do now is to choose two components of the energy-momentum tensor. For simplicity we choose as following

$$
\begin{aligned}
& \frac{i}{\omega} \delta \Theta_{t r}+\frac{F}{r\left(3 r h^{\prime}+2 \lambda\right)}=0 \\
& \frac{2}{\omega h} \delta \Theta_{t \theta}+\frac{i F}{h\left(3 r h^{\prime}+2 \lambda\right)}=0 .
\end{aligned}
$$

Since $\delta \Theta_{\mu \nu}=0$ in $4 d$ limit, Eq.(24) implies $F=0$ in the same limit. This is a boundary condition the authors in Ref. [4,13] used for the derivation of the $4 d$ effective potentials in the axial and polar perturbations. Thus Eq.(24) guarantees the potential derived by making use of this equation has a correct $4 d$ limit. Furthermore, Eq.(24) makes Eq.(23) reduce to the simple expressions, which enables us to derive the effective potential analytically.

Then Eq.(16) fixes the remaining non-diagonal component $\delta \Theta_{r \theta}$. Expressing $f_{1}, f_{2}$ and $g_{2}$ in terms of $g_{1}$ as following 


$$
\begin{aligned}
& f_{1}=-h g_{1}^{\prime}+\frac{3 r^{2} h^{\prime 2}+5 \lambda r h^{\prime}+2 \lambda h+2 \lambda^{2}}{r\left(3 r h^{\prime}+2 \lambda\right)} g_{1} \\
& f_{2}=i r\left[g_{1}^{\prime}+\frac{3 r^{2} h^{\prime 2}+2 \lambda r h^{\prime}-4 \lambda h}{2 r h\left(3 r h^{\prime}+2 \lambda\right)} g_{1}\right] \\
& g_{2}=-\frac{i r}{h} g_{1}
\end{aligned}
$$

and choosing $g_{1}$ as

$$
g_{1}=\left[(n+1) \frac{r_{H}}{r^{2} h^{\prime}}\right]^{1 / 2(n+1)}\left(\frac{2 \lambda r_{H}}{r\left(3 r h^{\prime}+2 \lambda\right)}\right)^{n / 2(n+1)},
$$

one can make a Schrödinger-like equation

$$
\left(\frac{d^{2}}{d r_{*}^{2}}+\omega^{2}\right) \hat{K}=V_{P}(r) \hat{K}
$$

where $r_{*}$ is defined as $d r / d r_{*}=h$ and

$$
\begin{aligned}
V_{P}(r)=\frac{h}{r^{2}\left(3 r h^{\prime}+2 \lambda\right)^{2}}\left[8 \lambda^{3}\right. & +\left(24 r h^{\prime}+8 h+2 r^{2} h^{\prime \prime}\right) \lambda^{2} \\
& +\left(18 r^{2} h^{\prime 2}+24 r h h^{\prime}+3 r^{2} h h^{\prime \prime}-3 r^{3} h h^{\prime \prime \prime}\right) \lambda \\
+ & \left.\left(18 r^{2} h h^{\prime 2}+9 r^{3} h h^{\prime} h^{\prime \prime}+\frac{27}{4} r^{4} h h^{\prime \prime 2}-\frac{9}{2} r^{4} h^{\prime 2} h^{\prime \prime}-\frac{9}{2} r^{4} h h^{\prime} h^{\prime \prime \prime}\right)\right] .
\end{aligned}
$$

When $h=1-\left(r_{H} / r\right)^{n+1}$, the effective potential (28) reduces to

$$
\begin{aligned}
& V_{P}(r)=h \frac{r^{2 n}}{\left[2 \lambda r^{n+1}+3(n+1) r_{H}^{n+1}\right]^{2}} \\
& \times\left[8 \lambda^{3}+2\left\{4-\left(n^{2}-9 n-6\right)\left(\frac{r_{H}}{r}\right)^{n+1}\right\} \lambda^{2}\right. \\
& -3(n+1)\left(\frac{r_{H}}{r}\right)^{n+1}\left\{n(n+6)-\left(n^{2}+12 n+6\right)\left(\frac{r_{H}}{r}\right)^{n+1}\right\} \lambda \\
& \left.+\frac{9}{4}(n+1)^{2}\left(\frac{r_{H}}{r}\right)^{2 n+2}\left\{n(n-2)+\left(n^{2}+8 n+4\right)\left(\frac{r_{H}}{r}\right)^{n+1}\right\}\right] \text {. }
\end{aligned}
$$

It is easy to show that $V_{P}(r)$ has a correct $4 d$ limit. But same problem arises as in the case of the axial perturbation. The general principles we used cannot fix the energy-momentum tensor completely. Thus we choosed two components in Eq.(24). The different choice may yield a different potential. Without knowing the exact nature of the matter on the brane it seems to be impossible to derive an unique effective potential. 
One may argue the situation may be improved if the Newman-Penrose formalism is applied. But there is also ambiguity in the Ricci terms which should be related to the energymomentum tensor. Thus same problem occurs even in the Newman-Penrose formalism.

In this letter the polar perturbation is examined when the spacetime background is a metric induced on the brane from the bulk Schwarzschild geometry. Since the projected background is not a vacuum solution of the Einstein equation, we tried to examine the behavior of the energy-momentum tensor under the polar perturbation from the general principles. As in the case of the axial perturbation, however, the general principles do not fix two components of the energy-momentum tensor. The suitable choice of these unfixed components yields an effective potential which has a correct $4 d$ limit. But the problem is the fact that different choice may yield a different potential. It is greatly nice if there is a physical mechanism which fixes all components of the energy-momentum tensor completely.

Acknowledgement: This work was supported by the Kyungnam University Research Fund, 2006. 


\section{REFERENCES}

[1] T. Regge and J. A. Wheeler, Stability of a Schwarzschild Singularity, Phys. Rev. 108 (1957) 1063;

[2] S. Chandrasekhar, The Mathematical Theory of Black Hole (Oxford University Press, New York, 1983).

[3] C. V. Vishveshwara, Scattering of Gravitational Radiation by a Schwarzschild Blackhole, Nature 227 (1970) 936.

[4] F. J. Zerilli, Effective Potential for even-parity Regge-Wheeler Gravitational Perturbation Equations, Phys. Rev. Lett. 24 (1970) 737.

[5] J. Polchinski, String Theory (Cambridge University Press, Cambridge, 1998).

[6] N. Arkani-Hamed, S. Dimopoulos and G. Dvali, The Hierarchy Problem and New Dimensions at a Millimeter, Phys. Lett. B429 (1998) 263 [hep-ph/9803315].

[7] L. Randall and R. Sundrum, A Large Mass Hierarchy from a Small Extra Dimension, Phys. Rev. Lett. 83 (1999) 3370 [hep-ph/9905221].

[8] F. R. Tangherlini, Schwarzschild Field in $n$ Dimensions and the Dimensionality of Space Problem, Nuovo Cimento 27 (1963) 636.

[9] R. C. Myers and M. J. Perry, Black Holes in Higher Dimensional Space-Times, Ann. Phys. 172 (1986) 304.

[10] H. Kodama and A. Ishibashi, A master equation for the gravitational perturbations of maximally symmetric black holes in higher dimensions, Prog. Theor. Phys. 110 (2003) 701 [hep-th/0305147].

[11] D. K. Park, Hawking Radiation of the Brane-Localized Graviton from the $(4+n)$ dimensional Black Hole Class. Quant. Grav. 23 (2006) 4101 [hep-th/0512021].

[12] E. Newman and R. Penrose, An Approach to Gravitational Radiation by a Method of 
Spin Coefficients, J. Math. Phys. 3 (1962) 566.

[13] L. A. Edelstein and C. V. Vishveshwara, Differential Equations for Perturbations on the Schwarzschild Metric, Phys. Rev. D1 (1970) 3514. 